\title{
Intelligent instrumentation: a quality challenge
}

\author{
H.J. Kohoutek \\ Hewlett-Packard Company, Colorado Computer Quality Department, Fort Collins, Colorado, U.S.A.
}

This is a reissue of a paper which appeared in ACTA IMEKO ACTA IMEKO 1988, Proceedings of the Proceedings of the $11^{\text {th }}$ Triennial World Congress of the International Measurement Confederation (IMEKO), "Instrumentation for the 21st century", 16-21.10.1988, Houston, pp. 337-345.

After a review and description of current trends in the design of electronic measurement and analytical instrumentation, changes in its application and use, and of associated quality issues, this paper deals with new quality issues emerging from the expected increase of artificial intelligence impact on system design and implementation strategies. The concept of knowledge quality in all its aspects (i.e. knowledge levels, representation, storage, and processing) is identified as the key new issue. Discussion of crucial knowledge quality attributes and associated assurance strategies suggests the need to enrich the assurance sciences and technologies by the methods and tools of applied epistemology. Described results from current research and investigation, together with first applications of artificial intelligence to particular analytical instruments, lead to conclusion that the conceptual framework of quality management is, in general, adequate for successful resolution of all quality issues associated with intelligent instrumentation.

Section: RESEARCH PAPER

Keywords: instrumentation design, measurement quality

Citation: H.J. Kohoutek, Intelligent instrumentation: a quality challenge, Acta IMEKO, vol. 3, no. 1, article 11, May 2014, identifier: IMEKO-ACTA-03 (2014)-01-11 Editor: Luca Mari, Università Carlo Cattaneo

Received May $1^{\text {st }}, 2014$; In final form May $1^{\text {st }}, 2014$; Published May 2014

Copyright: () 2014 IMEKO. This is an open-access article distributed under the terms of the Creative Commons Attribution 3.0 License, which permits unrestricted use, distribution, and reproduction in any medium, provided the original author and source are credited

\section{INTRODUCTION}

Regular reports on use of electronic measurement and analytical instrumentation, e.g. $[1,2]$, indicate that scientists and engineers plan to acquire more and more complex instruments (such as spectrophotometers, chromatographs, electron microscopes) and laboratory data management systems. To maximize the information gain from their application in physical sciences, life sciences, medicine, and engineering, a relatively high level of user knowledge and operational skill is required. This trend toward more complex instrumentation is accompanied by changes in the way professionals fulfill their various tasks [3]. We observe growth of time segments dedicated to study, learning, analysis, reasoning, and judgement in the structure of their jobs, with corresponding decline in data gathering, memorization, and physical activity. There is also a corresponding change in the generic structure of the instrument's block diagram [4]: From a simple measuring device dedicated to specific physical quantities, followed by transducers interfacing a generic electrical instrument, to current instrumentation (with both analog and digital electronic components) combined with data acquisition and processing. The architecture of contemporary instrumentation permits implementation of complex and abstract models of applicable physical, chemical, and information transformation processes. Similar change is observable in the modes of instrumentation controls from mostly manual (allowing operators to adjust the set-up) to stored programs and feedback algorithms, allowing steadily increasing levels of automation in sample handling, repetitive measurements, and self-test. Another of the key changes in test and measurement instrumentation is the evolution of small multifunction products (usually an instrument-on-a-card) able to replace an entire rack [5].

The dominating technical issues being addressed by contemporary designers are concentrated around:

- Requirements for real time measurements [6], reflected in increases of rates of data acquisition, speed of $A / D$ conversion, rates of data processing, careful selection of data format, interrupt priorities, etc. Major unsolved problems remain in the areas of multinodal matrix data acquisition and processing of unstructured inputs.

- Assessing the strategic impact of personal computers [7] which are strengthening their foothold in the field of electronic instrumentation. Although PC-based instrumentation does not provide anything substantially new, it handles many data related tasks less expensively and 
with more flexibility than dedicated processors. Their memory with centrally stored programs and data permits efficient record keeping, cooperation of multiple instruments or measurement processes, and communication. This frees the user from many routine laboratory and reporting chores.

- Interfacing, stemming from the availability of data processing equipment and need for at least laboratory-wide networking.

All this is a contemporary reflection of the intuitively obvious and routine search for more capability (on-line measurements, automation of sample handling, ...), higher performance (detection limits, sensitivity, selectivity, signal-tonoise ratio, speed, ...), ease of use, and lower price. In more specific terms the overall goals of new developments in the various types of instrumentation are:

- Enhanced productivity and creativity of the analyst and experimenter,

- increased information content of experiments,

- optimized choice of experiment conditions,

- multidimensional and multiparametric measurements, and

- an integrated, highly automated laboratory.

In general, these instrument design trends [8] seem to be driven by:

- Need for wide repertoires of techniques and methods in a single laboratory, which is expected to be satisfied by implementations that preclude the prohibitive cost of a large number of dedicated instruments.

- Recognized power of software leading to more software controlled instrumentation [9] and to blending of computations and measurements, in these systems the instrumentation parts are expected to be fixed, while the software assures needed flexibility and defines how the individual components behave together. This conceptual arrangement permits radical changes in the experiment to be accomplished with minimal hardware changes, In addition, software driven instrumentation opens door for self-tuning, optimization, self-diagnostics, integration into the overall laboratory environment, etc.

- Availability of new and exotic technologies, such as fiber optics and microfabrication, offering an enormous range of opportunities for chemical microsensors (e.g. CHEMFETs, microdielectrometers, surface acoustic wave sensors), multiwavelength measurements, portability, and cost reduction $[10,11]$.

The overall pace of changes along these recognized trends will probably quicken and be more dramatic after the introduction of research results of Artificial Intelligence (AI) into routine instrument design considerations (block diagram of an intelligent instrument is presented in Figure 1). Experience currently being accumulated in the design of a wide variety of expert systems, complementing the well published work on DENDRAL, MACSYMA, PROSPECTOR, MYCIN, etc., will not only impact the instrumentation design, but will change the jobs of both instrument designers and instrument users [12], and highly probably, create new Artificial Intelligence technology based jobs in support areas. The expected result will be intelligent instrumentation which will be able to solve problems or produce answers instead of data [13].

The overall objective of introducing AI into instrumentation is to free the professional experimenter from unnecessary involvement with minute implementation details of the experiment and give him or her intelligent assistance in data interpretation, experiment evaluation and, ultimately, experimentation and instrumentation control according to embedded knowledge. Results from current research and application implementations, e.g. [14, 15], are presenting a convincing argument about the reality of an expected major impact by AI on the new generation of instruments. Embedded artificial intelligence could easily become the dominant design and implementation strategy for 21 st century instrumentation.

\section{GENERIC QUALITY ISSUES IN CONTEMPORARY INSTRUMENTATION}

Measurement systems performance, like all technical equipment performance, is a result of sometimes complex interactions among the equipment, the operator, the procedures used, and the environment in which uninterrupted performance is expected. Quality management must address all these factors, because they all can be the root cause of imperfect performance representing itself as a detect, an error, or a failure [16].

The quality characteristics of the measurement process have developed around the concepts of precision, the degree of mutual agreement of independent measurements yielded by repeated

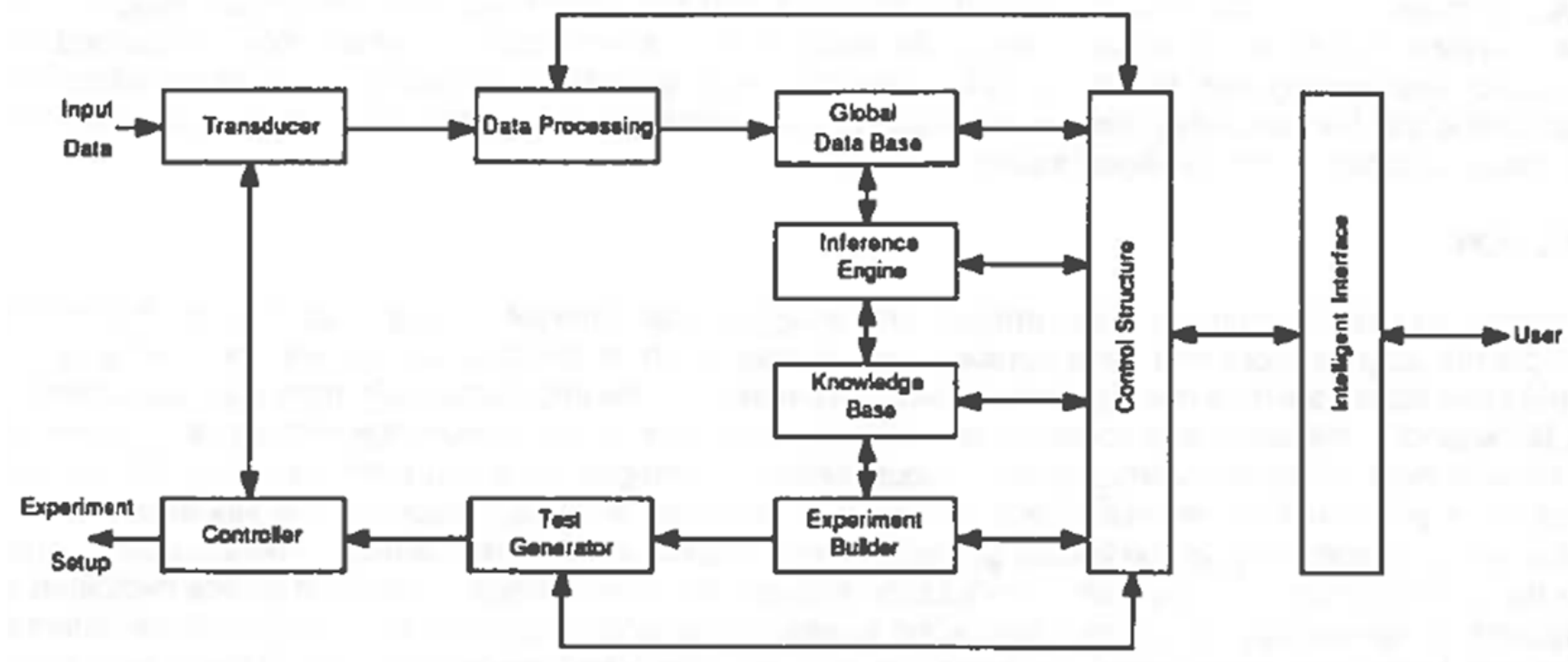

Figure 1. Block diagram of an intelligent instrument. 
applications of the process under specified conditions; and accuracy, the degree of agreement of such measurements with the true value of the magnitude of the quantity under consideration [17]. The concept of precision lends itself to a rigorous mathematical treatment by methods of statistical analysis. For accuracy, unfortunately, there does not exist any single, comprehensive, and satisfactory measure, and in some particular situations the concept itself can become illusive. Here statistics can only help, e.g. with tools such as mean square error, as proposed by Gauss himself, measurement bias, systematic error, and measurement uncertainty. But even with these tools the steps for analyzing assignable and unassignable causes of error are plagued with many difficulties.

To satisfy the statistical character of both concepts multiple measurements are needed, which leads to an empirical concept of the repeatability of a measurement. Repeatability implies the same measurement process and the same method of measurement. Considered more fully it should also include the same observer, operators, auxiliary equipment, and equipment calibration status. In addition, it should be based on random sampling which takes into account a reasonable range of circumstances.

Out of the wide variety of measurement equipment quality attributes the ones closest to measurement process quality are performance linearity over the effective range, and instrument stability. Linearity (actually the non-linearity or lack of linearity) is usually conveyed by statements about how the signal deviates as it spans the range of measurements. Linearity of an instrument is generally determined as the result of a calibration test in which only limited number of values is determined for a system in steady state. Only rarely will the same relation apply for dynamic states because the storage parameters will alter the instantaneous transfer characteristics according to the signal amplitude and history of previous signal excursions. Pressure gauges, accelerometers, thermometers, etc. will give very different values for instantaneous and steady state measurements. The best expression of an instrument linearity is via the calibration curve showing deviations from the best straight line.

Drift is a system characteristic, sometimes understood to be synonymous with the term stability, that characterizes how a system variable, which is intended to be constant, varies with time. It also conveys information about very-low-frequency response of the instrument to the measured. Drift characteristics are usually specified from the reference point (defined at $25^{\circ} \mathrm{C}$ ) to the extremes of the working range, expanded by safety margins.

Linearity and stability, traditionally associated with the notion of instrument quality, are mostly a direct function of design choices and goals. Other typical design objectives, intended to minimize deterioration due to time or environmental stresses, represent additional product quality attributes which are achieved by proper system structure, component selection, adequate design margins, and built-in redundancy. These attributes are most often known as:

- Reliability, the probability of a system's successful function over a given mission period [18].

- Environmental ruggedness, defined by a class of environmental stresses under which proper functionality is guaranteed.

- Electromagnetic compatibility, given by the energy levels, directions, and frequencies of radiated and conducted emissions and by the instrument susceptibility to external electromagnetic fields.
- Safety level or class, indicating the risks of explosion, fire, or other hazards caused by potential internal defects or failures.

- Maintainability [19], which is the probability that, when maintenance action is performed under stated conditions, a failed system will be restored to operable condition within a specified total down time.

Levels of these product quality characteristics, required for business success and customer satisfaction, are given by users' expectations and set by market conditions, competitive products performance, state-of-the-art of assurance technologies, and by government regulations or international standards. In some situations the overall economic value of these characteristics can be expressed by cost of ownership, which takes into account values of vendor's goodwill shown in warranty period and conditions, cost of support and service contracts, etc.

To the product quality characteristics mentioned above we must add the classical expectations of products free from defects at delivery, compliant to product specifications and data sheets, and evidencing satisfactory level of manufacturing quality controls, adequate workmanship skills, and attention to detail.

Advances in computing technologies and the related lower cost of computing found their applications in many fields, including analytical, control, and measurement instrumentation. Currently the major part of electronic instrumentation is software driven, which brings into focus issues of software quality. The quality of the whole measurement system may, to a significant degree, be dependent on the quality of the code. Because computer software is a logical rather than a physical system, it has different characteristics [20], e.g.:

- it does not wear out,

- maintenance often includes design modifications and enhancements, and

- there is no significant manufacturing activity involved.

There is also not very much well structured industrial experience to significantly indicate preferable engineering design strategies. Many attempts to define software quality characteristics with associated metrics, criteria, and contributing factors have been made, e.g. [21, 22, 23], but only a few members of the proposed family of quality attributes are universally accepted, mainly:

- Reliability, meaning a measure of the frequency and criticality of unacceptable program behavior under permissible operating conditions;

- Correctness, now inconclusively demonstrated by validation testing which provides some level of assurance that final software product meets all functional and performance requirements. There is some hope that, in the future, programs will be infallibly proven to be correct. Some tools for automated proofs are beginning to emerge from the research work in artificial intelligence.

- Maintainability, which may be defined qualitatively as the ease with which software can be understood, corrected, adapted, and enhanced. Software maintainability is a difficult term to quantify, but Gilb [24] proposed a number of measures relating to the maintenance effort.

- Ease of use assures that the job or task will be successfully completed by personnel at any required stage in system operation within a specified time. Usability characteristics are based on observations and understanding of operator 
behavior, limitations of body characteristics, and reaction times.

Design approaches and assurance techniques for these quality attributes have not stabilized yet and vary widely from vendor to vendor.

In the commercial world, as in science and engineering, these described measurement system quality characteristics are complemented by the ever increasing need to demonstrate that the total measurement uncertainty is sufficiently small to meet users requirements. This measurement assurance, in reality a quality control of measurements, is based on redundancy built into the measurement scheme. This redundancy is usually represented by repeated measurements of a stable standard. Measurement assurance is then obtained when verifiable limits of uncertainty have been demonstrated, and measurement process quality control program exists in real time to monitor its performance $[25,26]$.

\section{QUALITY ISSUES UNIQUE TO INTELLIGENT INSTRUMENTATION}

- The definition of quality, which is currently oriented toward conformance to specification. It needs to be replaced by a sociometric definition, which perceives and eventually measures it as a multiattribute product characteristic to be designed in;

- The value of quality, now primarily beneficial to the manufacturer via reduced production and support cost, must be recognizable directly at the product level in both classical forms (value in exchange and value in use). This will allow quality to be properly priced, and appreciated as a source of revenue;

- The role of the quality department, where quality specialists and managers must shun being critics of manufacturing processes and become contributing partners with all line functions.

We understand the fact that scientific research, as well as control and measurement processes are, by nature, processes of goal oriented learning, as evident from the similarity of flow diagrams of basic process steps shown in Figures 2A, B. The key objective in making these processes as efficient as possible is frequently achieved by applying scientific analytical instrumentation. Further, considering that the learning process itself is a feedback loop between inductive formation of hypotheses, based on accumulated data, and collection of new data according to deductively proposed experiments, we can see that classical instrumentation plays a major role in data gathering and analysis. However, intelligent instrumentation is expected to bring new (expert) levels of efficiency into the

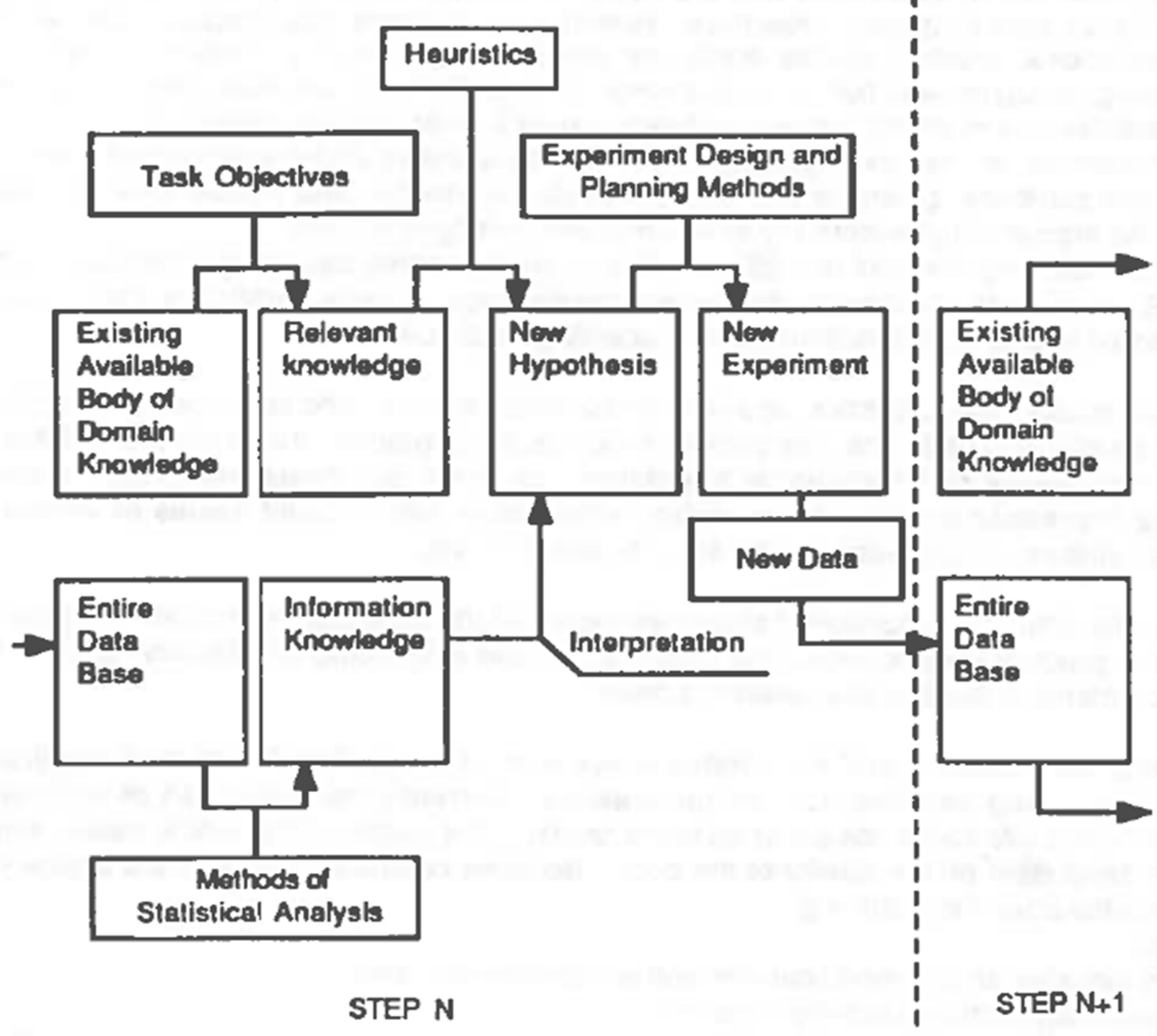

Figure 2A. The process of experimentation as iterative learning. 


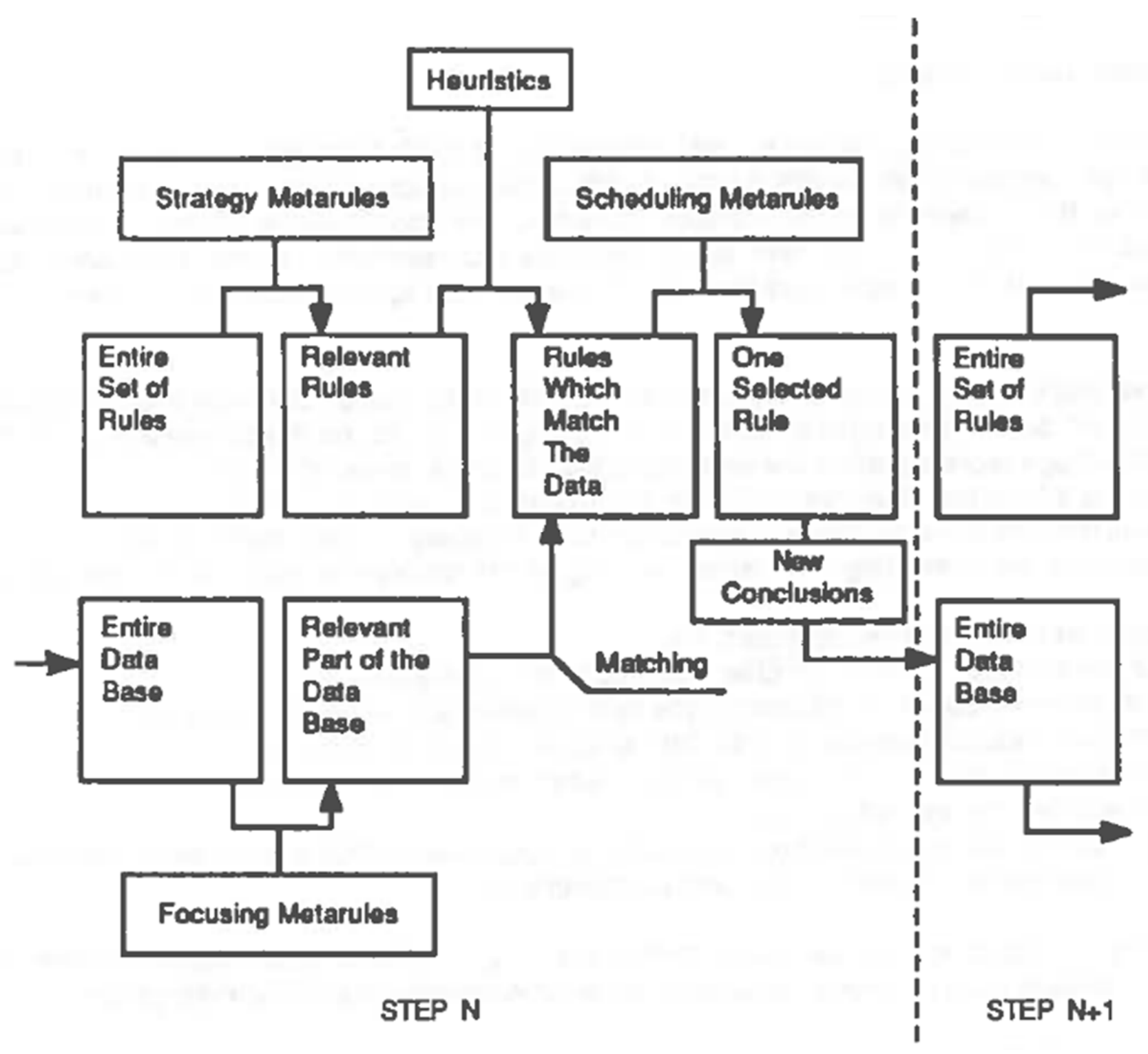

Figure 2B. Event-driven control scheme of a general problem solver.

processes of experimental data interpretation, new hypotheses forming, and designing of new experiments.

Recognition that all the terms, such as data, information, rules, metarules, heuristics, etc. used in presented flow diagrams, represent different forms of knowledge, brings us to knowledge as the central point of the whole held of artificial intelligence perceived, from the practical viewpoint of the electronic industry, as knowledge-based problem solving by computers. Also the empirical and intuitively self-evident observation that the competence, and by that the value, of any intelligent system strongly correlated to the amount and quality of the embedded knowledge, leads us to the central unique quality issue of intelligent instrumentation: the issue of knowledge quality.

\subsection{Knowledge Quality}

The factual part of our knowledge comes mostly in the form of scientific and technical data in three broad classes [27]:

- Repeatable measurements on well defined systems (class A); where quality assurance methodologies recommend using data only from reliable sources such as National Standard
Reference Data System (NSRDS) coordinated by the U.S. National Bureau of Standards.

- Observational data, often time or space dependent (class B). The most effective way to assure quality of class B data by careful maintenance and calibration of the measuring instruments prior to data acquisition. Recording and preservation of all required auxiliary information is also important.

- Statistical data (class C); here quality control strategy, based on Probability Theory, is a more difficult task, often hindered by disagreements in definitions and terminology.

One of the greatest benefits of the recent advances in graphics software is increased control over the quality and completeness of experimental data by allowing for its visual inspection via windowing, 3-D modelling, and simultaneous processing of alphanumerical and graphical information. Improvements have reached the point where virtually any level of detail from multiple measurements can be observed on the screen and functionally used to enhance data quality.

Also more frequent acceptance of the need for systematic skepticism and openness to alternative modes of interpretation in data analysis is now leading to application of statistical 
methods of exploratory data analysis. These methods reflect recognition of the importance of graphical representation for visual inspection of unprocessed data, which should precede formal statistical calculations even when the latter is the desired end product. This approach opens wider ranges of alternative explanations and allows the researcher to be open to unexpected possibilities, particularly while working with weak theories.

Assuring quality of higher level knowledge, which is being engineered today and will be embedded in intelligent instrumentation (e.g. definitions, taxonomies, discrete descriptions, constraints, deductive algorithms), presents many epistemological challenges. The philosophical questions about the reliability of scientific knowledge, by themselves a serious intellectual issue, will not significantly impact the design of intelligent instruments. This is so because, in majority of expected applications, the knowledge and application domains will be sufficiently narrow and well defined. This will permit quality assurance to concentrate on issues of unambiguity, consistency coherence, visibility and acceptability of perceived patterns, and methods of justification and validation.

A completely new set of quality issues is associated with the problems of knowledge base updates and knowledge maintenance. Quality assurance methods here might be simplified by the presence of metaknowledge or metarules controlling automatic learning in embedded knowledge bases.

\subsection{Quality of Knowledge Representation}

The pragmatic approach to embedding knowledge into programs that exhibit intelligent behavior is focused on developing schemes for knowledge representation which permit manipulation of specialized data structures to obtain intelligent inferences. In general, the knowledge representation schemes are combinations of data structures and interpretive procedures. The most frequently used formalisms are: state/space representation, formal logic, procedural representation, semantic nets, production systems, scripts, and frames. There are also special techniques for visual scene and speech representation.

The issues of form and notation have occupied most previous quality discussions. Currently it is recognized that the issue of what could or could not be done with a representation is more important. So, on the conceptual level, the most important quality attributes of knowledge representation are associated with its three adequacies [28]:

- metaphysical, assuring a form that does not contradict the character of facts of interest,

- epistemological, which guarantees that the representation can practically express the facts, and

- heuristical, that assures the reasoning processes, leading to the problem solution, are expressible in contemplated language.

Many authors [29] proposed more practical attributes, e.g.:

- modularity, which allows adding, deleting, or changing information independently,

- uniformity, to assure understandability to all parts of the system achievable via rigid structures,

- naturalness, to reflect the ease of expressing important kinds of relevant knowledge,

- understandability, the degree to which the representation scheme is clear to humans,

- modifiability, which assures context independence.
Studies of semantic primitives and established representational vocabularies [30] lead to other attributes such as finitude, comprehensiveness, independence, noncircularity, and primitiveness.

A completely new quality assurance issue arises when problem solving algorithms require representation change midstream. Representation transformations must be then evaluated in terms of isomorphism and homomorphism.

\subsection{Knowledge Base Quality Issues}

Technical implementations of knowledge bases are benefiting from the design for quality methodologies of data bases, which culminated in fault-tolerant designs. New quality issues, emerging from the special characteristics of relational data bases and current experience with knowledge bases, are bringing into focus needs of assuring:

- Protections against interferences among the subsets of a knowledge base. This is complicated by the environment of laboratory-wide networks which do not have a central operating system. Current "design for quality" research results tend toward making transactions atomic, which could adversely affect the transaction time;

- Protections against new aspects of pattern sensitivity;

- Transparency of structural characteristics, a difficult task in conditions of high structural complexity; and

- Already mentioned knowledge maintenance quality controls. First attempts to formulate adequate assurance strategy for knowledge maintenance have already taken place, as described e.g. in [31], providing prespecified set of control metarules, production rules formalism, and tools for sensitivity analysis, execution tracing, and explanations. But effective implementation of these strategies will be complicated by the problems of scale, speed, and system complexity.

The importance of methods for validation of system's knowledge base and evaluation of its quality is also self-evident, but their development is a very difficult task.

\subsection{Quality of Knowledge Processing}

The basic set of knowledge processing algorithms consists of search processes, deductions, inductive inferences, and learning.

- Search algorithms, not significantly different from other algorithms implemented by computer programs, do not present new or unique quality problems. The basic questions here still relate to implementation correctness and its demonstrated verification levels. Some new challenges may be found in implementation of associative searches, but even here, the main issue is correctness.

- Deductions are basically chains of statements, each of which is either a premise or something which follows from statements occurring earlier in the chain. Mechanization of deductive processes based on predicate logic must, beside the issue of direct correctness, take into account needs for the deduction's visibility, ease of inspection, backward traceability etc., to improve solution credibility and to allow for correctness verification.

- The most difficult task for knowledge processing quality assurance arises when we depart from categorical (or deterministic) reasoning. Statistically based inferences, or inferences based on social judgement are plagued with errors of insufficient evidence, illusory correlations, 
circumstantial prompting, belief perseverance in the face of contrary evidence, etc. Research into application of epistemology to solve these types of problems is only beginning. But we do not expect these problems to be encountered during the early generations of intelligent instrumentation.

- Automated learning systems' performance can be measured by the rate of knowledge compilation. The quality of such a system is usually described by the system's generality (the ability to perform successfully in novel situations), its ability to discriminate between critical and peripheral information, its ability to automatically restrict the range of acquired knowledge applicability, and its ability to control the degree of interest of inferences in order to comply with principles of significance and goal satisfaction.

But the fundamental conceptual problem of controlling quality of knowledge processing will be resolved only if the problem of adequate modelling of the categorical structure of the thinking process will be resolved.

\section{OTHER QUALITY ISSUES ASSOCIATED WITH THE INTELLIGENT INSTRUMENTATION}

In many aspects the intelligent instruments will be implementations of an advanced generation of the current software driven instrumentation. Because of this fact all the traditional software quality issues will be still present and will have to be addressed. The aspects of the new, higher level of intelligent human interface will bring into the field of instrumentation quality issues currently associated with advanced computing and engineering workstations. The most important seem to be the:

- Quality aspects of the control language; in the field of intelligent instrumentation the interface and control language will have to provide convenient means for implementation of a variety of experimenting strategies and designs. It must also act as a vehicle of experimenter's thought, and via local area networks allow communication of higher level concepts between experimenters. The minimum quality requirements will have to cover aspects of ease of programming, error handling, automatic backtracking, machine independency of compilers, and level of standardization. Similar requirements will apply to languages designed specially for manipulating knowledge bases.

- Quality of human interface, which must permit the instrumentation user to concentrate fully on his experiment, without being distracted by the uniqueness of the applied computational technology. Human interface design strategies will have to go beyond the traditional ergonomic and intuitive approaches to design for system friendliness. They must address the physical, physiological, psychological, and intellectual aspects at the experimenters job and personality. Solutions must be based not only on anthropometry but be developed in the context of the measurement system's lexical, syntactic, and semantic requirements. There is a growing recognition of the need for serious scientific experimentation which will permit both the designer and cognitive psychologist to significantly improve current approaches to human-instrument interface designs.

- Quality aspects associated with new computing architectures and increased levels of parallelism.
- System testability and diagnosis; the growing complexity of the instrumentation, its real-time performance, which will make accurate repetitions of the computational and reasoning processes impossible due to asynchronisms and new levels of freedom for internal intelligent controls, will make these tasks especially difficult but nevertheless extremely important for future fault-tolerant designs.

Despite expected software dominance there still will be enough hardware in the next generation of instrumentation to keep us worrying about the traditional quality issues associated with product safety, regulatory compliance, environmental ruggedness, reliability, maintainability, supportability, etc. Some new quality issues will emerge with new families of VLSI components. The increased levels of their complexity and density will challenge our ability to control qualify of all materials involved in processing, the dimensions of manufactured structures in and below the one micron range, and the many process variables needed to achieve yield economy and final product quality.

The expansion of intelligent instrumentation, expected in 1990's, will be complemented by maturing of some sensor technologies currently being introduced. The new families of sensors solve one set of problems but open risks in other areas of which the quality assurance community must be aware, e.g.:

- Optical sensors for chemical analysis are an answer to problems of electrical interference, reference electrodes, physical contact with the reagents, and need for multiwavelength measurements. But to ensure the highest possible quality of their application, care must be taken to prevent background light interference, the possibility of photodegradation of reagents, to assure long term stability, etc. The sources of potential error must be identified and compensated for.

- Electrochemical sensors, where developments are moving away from potentiometric to amperometric sensors. These changes are bringing challenges to techniques of surface modification control required to achieve wanted selectivity, to compensation methods for dealing with increased sensitivity and to design for reliability. For CHEMFETs, especially, challenges will appear in areas of stability and encapsulation technologies.

The fundamental quality issues and strategies for assurance of these new families of sensors revolve around:

- the two concepts: compound confirmation and compound identification, as well as

- the practical need to avoid application surprises, such as interference in sample matrix effects, errors at the reference point, etc.

\section{RESULTS OF CURRENT RESEARCH AND APPLICATIONS}

Reports regarding results of AI application in support of instrumented diagnostics and troubleshooting are many. Manufacturers, including IBM, Digital, Tandem, Prime, AT\&T, General Electric (for review see e.g. [32]), have devised remote diagnostic expert systems to analyze hardware problems in the field. In 1984 major breakthrough by Dr. L.F. Pau at Battelle Memorial Institute, Switzerland, in development of a set of about 400 application independent metarules for diagnostic expert systems, provided new strategy for both reduction of design cost and increase of capability via a model of a circuit network which describes many types of real electronic faults. Also the proliferation of CAE tools and workstations has a far reaching impact on the productivity of development and ease of 
support of maintenance related expert systems. Other software based expert system-building tools, such as automated reasoning tool ART [33], permits the designer to develop the necessary knowledge base, reflecting his experience. These tools often use frames to represent knowledge about objects of interest, along with their attributes. Agenda or menu windows indicate the rules fired together with facts that initiated them. These and command windows, which trace program execution step-by-step, simplify both debugging and use of the system by illustrating the reasoning process flow.

Less visible, but significant, are the results of the still infrequent applications of AI to upgrade performance of analytical instruments. One of the key reasons for this slow progress is that the majority of known successful projects has been implemented on large computers, often unavailable in current laboratory settings.

A microcomputer based expert system for enhancement of electroencephalogram evaluation [15] has demonstrated the usefulness of AI methods in biomedical instrumentation. This system provides on-line evaluation with performance equal to that of a large computer. The most important information is being extracted from the frequency domain, using FFT for spectral estimation, Hanning windows to reduce spectral leakage, and forty-three assembly language coded rules for basic classification into either normal or abnormal categories. The disadvantage of the present system lies in the need to have the user provide the complete control strategy, i.e. the selection of relevant rules and sequence of their implementation.

The development of a totally computerized triple quadrupole mass spectrometer, with prototype knowledge based instrument control [14], is a necessary step toward the goal of intelligent TQMS instrumentation. It has the ability to apply structured stored experience, to use power of automatic reasoning to control its own behavior, and to respond to novel situations and new problems. The knowledge including control heuristics is represented in the form of productions (rules). Resident, AI guided calibration program assures self-adaptive feedback control process for real-time optimization or tuning of the data acquisition process.

Studies of data acquisition, knowledge representation, reasoning algorithms, and interpretation of results, applied, e.g., to chromatographic data for the diagnosis of spontaneous bacterial peritonitis [34], indicate that current results can be extended to other applications and could lead to automated intelligent decision systems.

Honeywell System's FLEET/DRAS project [35] demonstrated the feasibility of applying expert system technology to automation of data reduction, analysis, and detection of anomalies.

Although incomplete, work done in investigation and assessment of the mutual relationship between artificial intelligence and assurance sciences and technologies, has identified the key quality strategies necessary to address the new, knowledge quality related issues [36]. These strategies are based on creative application of current assurance expertise enriched by methods of applied epistemology. As described in previous chapters major progress has been achieved in:

- controlling quality of the factual part of embedded knowledge;

- identification of adequacy (metaphysical, epistemological, and heuristical) as the most important quality attribute of a knowledge representation;
- strategies for fault-tolerant design of data and knowledge bases; and

- key architectural guidelines for intelligent human interface design.

The key quality assurance technologies and skills for the manufacture of key VLSI components, which are expected to be fundamental for the intelligent instrumentation hardware, are identified and in place [37].

New sensor technologies, new non-von Neumann architectures, new system parts and functions, as well as support of knowledge quality assurance, will require additional intensive applied research and engineering work by teams of experts from both the R\&D and quality communities.

\section{SUMMARY AND CONCLUSIONS}

The emergence of successful practical implementations of the research results in artificial intelligence is beginning to impact conceptual thinking about the new generation of electronic measurement and analytical instrumentation. Because the competence, and by that the value of an intelligent instrument, will always be strongly correlated to the amount and quality of the embedded knowledge, the issue of knowledge quality is beginning to form the cornerstone of the new concern of quality management. To assure success of quality programs, methods of applied epistemology must be employed. Only in this way can the problems of quality at all levels of knowledge, its representation, storage, and processing be solved. The key quality assurance strategies addressing these knowledge related quality attributes have been identified.

New quality issues are also emerging because of the increased levels of hardware parallelism, computational speed, system complexity, and new sensor technologies. Traditional issues of hardware and software quality, as well as issues of support and vendors goodwill, remain substantially unchanged.

Current research and application results indicate that artificial intelligence must be considered by instrumentation designers in their search for more capability, higher performance, ease of use, and lower price. They even suggest that embedding artificial intelligence into the measurement and analytical instrumentation could easily become the dominant system design and implementation strategy for the 21 st century.

The conceptual framework of current quality assurance, if enriched by methods and tools of applied epistemology as indicated, seems to be generally adequate to address the new issues associated with knowledge quality and intelligent user interface.

\section{REFERENCES}

[1] C.J. Mosbacher, Use of analytical instruments and equipment, Research \& Development, Feb. 1985, pp. 174-177.

[2] C.J. Mosbacher, Use of analytical instruments and equipment, Research \& Development, Feb. 1986, pp. 90-93.

[3] F. Hayes-Roth, The machine as partner of the new professional, IEEE Spectrum, Jun. 1984, pp. 28-31.

[4] C.H. House, Perspectives on dedicated and control processing, Computer, Dec. 1980, pp. 35-49.

[5] G. Sideris, Traditional products get squeezed by newer gear, Electronics, Feb. 1987, pp. 89-93.

[6] J.G. Liscouski, Selecting instrument interfaces for real-time data acquisition, Analytical Chemistry, 1982, 54 (7) p. 849.

[7] F. Guteri, Instrumentation (Technology 1985), IEEE Spectrum, Jan. 1985, pp. 65-67.

[8] W.E. Terry, Instrument advances will be an intelligent progression, Electronic Business, Dec. 1985, pp.137-138. 
[9] M.L. Salit, M.L.Parson, Software-driven instrumentation; the new wave, Analytical Chemistry, 1985, 57 (6) p.715.

[10] V. Berry, Liquid chromatography at the 1986 Pittsburg Conference; Detectors, American Laboratory, May 1986, pp. 36-48.

[11] H. Wohitjen, Chemical microsensors and microinstrumentation, Analytical Chemistry, 1984, 56 (1), p.87.

[12] P. Wailich, The engineer's job; it moves toward abstraction, IEEE Spectrum. Jun. 1984, pp. 32-47.

[13] J. Sztipanovits, J. Bourne, Design of intelligent instrumentation, Proc. of the First Conference on Artificial Intelligence Applications, IEEE Computer Society, Dec. 1984, pp. 490-495.

[14] C.M. Wong, R.W. Crawford, J.C. Kunz, T.P. Kehler, Application of artificial intelligence to triple quadrupole mass spectrometry, IEEE Trans. NVC1. SC1, Vol. NS-31, No. 1, Feb. 1984, pp. 804-810.

[15] L. Baas, J.R. Bourne, A rule-based microcomputer system for electroencephalogram evaluation, IEEE Trans. Biomed. Engr., vol. BME-31, No. 10, Oct 1984, pp. 660-664.

[16] F.G. Peuscher, Design and manufacture of measurement systems, Handbook of Measurement Sciences, Vol. 1, ch.28, pp.1209-1215 John Wiley \& Sons Ltd, 1983.

[17] H.H. Ku, Editor: Precision measurements and calibrations, NBS special publication 300, Vol. 1, Feb. 1969.

[18] M.L. Shooman, Probabilistic reliability, McGraw-Hill Book Co., New York, 1968.

[19] J.G. Rau, Optimization and probability in systems engineering, Van Nostrand Reinhold Co., New York, 1970

[20] R.S. Pressman, Software engineering, McGraw-Hill Book Co., 1982.

[21] B. Boehm, et al., Characteristics of software quality, North Holland Publishing Company, 1978.

[22] T. McCall, et al., Factors in software quality, GE-TIS-77CISO2, General Electric Company, 1977.

[23] W. Curtis, Management and experimentation in software engineering, Proc. of the IEEE, Vol. 68, No. 9, Sep. 1980.

[24] T. Gilb, A comment on the definition of reliability, ACM Software Engineering Notes, Vol.4, No. 3, July 1979.
[25] J.W. Locks, Measurement assurance and accreditation, Proc. of the IEEE, Vol. 74, No. 1, Jan. 86, pp. 21-23.

[26] R.M. Judish, Quality control of measurements - Measurement assurance", Proc. of the IEEE, Vol. 74, No 1, Jan 86, pp. 23-25.

[27] D. Lide Jr., Critical data for critical needs, Science, Vol. 212, No. 4501, June 1981, pp. 1343-1349.

[28] J. McCarthy, P.J. Hayes, Some philosophical problems from the standpoint of artificial intelligence, Readings in Artificial Intelligence, Tioga Publishing Co., Palo Alto, California,1981, pp. $431-450$.

[29] A. Barr, E. Feigenbaum, Editors, Handbook of artificial intelligence, Vol I, Chapter III, pp. 193-194.

[30] Y.A. Wilks, Methodological questions about artificial intelligence; approaches to understanding natural language, Journal of Pragmatics, Vol. 1 (1977), pp. 69-84.

[31] P. Politakis, S.M. Weiss, Using empirical analysis to refine expert system knowledge bases, Artificial Intelligence, Vol. 22, No 1 (1984), pp. 23-48.

[32] H. Lahore, L. Gozzo, Artificial intelligence application to testability, Proc. of Annual Reliability and Maintainability Symposium, 1985, pp. 276-282.

[33] C. Kalme, AI tool applies the art of building expert systems to troubleshooting boards, Electronic Design, Apr. 1986, pp. 159166.

[34] M.E. Cohen et al., Knowledge representation and classification of chromatographic data for diagnostic medical decision making, pp. 481-486.

[35] W.K. Utt et al, An expert system for data reduction, Proc. of the 2nd Conference on Artificial Intelligence Applications, Miami Beach, Florida, USA, 1985, IEEE Computer Society / NorthHolland, pp. 120-124.

[36] H.J. Kohoutek, Quality issues in new generation computing, Proc. of the International Conference on Fifth Generation Computer Systems, ICOT, 1984, pp. 695-670.

[37] H.J. Kohoutek, Quality assurance technologies for VLSI manufacturing, Quality and Reliability Engineering International, vol. 3 (1987), pp. 107-126. 